\title{
Espondilolistese degenerativa: avaliação da qualidade de vida dos pacientes submetidos a tratamento cirúrgico
}

Degenerative spondylolisthesis: life quality evaluation of patients submitted to surgical treatment

Luciano Miller Reis Rodrigues' ${ }^{1}$ Fabrício Hidetoshi Ueno' ${ }^{1}$ Edgar Valesin Santiago Filho', Rodrigo Junqueira Nicolau', Tatiana Dias de Carvalho' ${ }^{1}$, Adriano Masayuki Yonezaki ${ }^{1}$, Rafael Carvalho Jorgetti ${ }^{1}$

\begin{abstract}
Resumo
Objetivo: Avaliar a mudança da qualidade de vida dos pacientes com espondilolistese degenerativa após tratamento cirúrgico (descompressão e artrodese posterolateral associadas com o uso de parafusos pediculares). Método: Trinta pacientes com espondilolistese degenerativa foram operados de janeiro de 2005 a março de 2007 tendo sido submetidos à descompressão com artrodese posterolateral com uso de parafusos pediculares. Todos os pacientes foram avaliados pela escala visual analógica de dor (EVA), pelo Índice de Incapacidade de Oswestry e pelo Questionário de Roland-Morris. Depois desse período, o método de Wilcoxon foi utilizado para a análise estatística da qualidade de vida dos pacientes após os procedimentos cirúrgicos. Resultados: Na escala analógica de dor houve uma melhora de $86 \%$ da dor. Na classificação de Oswetry, notamos uma melhora de $50 \%$ dos casos e utilizando o método de Roland-Morris, constatamos uma melhora de 22,91\%. Conclusões: 0 tratamento cirúrgico com descompressão e artrodese posterolateral associado à instrumentação pedicular possibilitou melhora a qualidade de vida dos pacientes com espondilolistese degenerativa.
\end{abstract}

Palavras-chave: Espondilolistese/cirurgia; qualidade de vida.

\begin{abstract}
Objective: To evaluate changes in quality of life of patients with degenerative spondylolisthesis after surgical treatment (decompression and posterolateral lumbar fusion with associated pedicular screws). Method: Between January 2005 and March 2007, 30 patients with degenerative spondilolisthesis who underwent decompression and posterolateral fusion with associated pedicular instrumentation were evaluated. All patients were assessed before and after surgery by the visual analog scale (VAS), Oswestry Disability Index and Roland-Morris Disability Questionnaire. After this period a statistical analysis by Wilcoxon test was done to evaluate changes in quality of life in these patients after surgical procedures. Results: Considering VAS, we assessed a pain improvement in all patients of $86 \%$. Using the Oswestry Disability Index we noted an improvement of more than $50 \%$ of cases. About Roland-Morris Questionnaire this study showed 22,91\%. Conclusions: Decompression with posterolateral lumbar fusion associated with pedicular instrumentation improves quality of life in patients with degenerative spondylolisthesis.
\end{abstract}

Keywords: Spondylolisthesis/surgery; quality of life;

Recebido: 22/5/2009

Revisado: 25/11/2009

Aprovado: 28/12/2009

Trabalho realizado no Hospital Estadual Mário Covas de Santo André (SP), Brasil.

Grupo de Coluna da Disciplina de Ortopedia e Traumatologia da Faculdade de Medicina do ABC, Santo André (SP), Brasil.

Endereço para correspondência: Luciano Miller Reis Rodrigues - Rua Borges Lagoa, 1065, cj. 40 - CEP 04038002 - São Paulo (SP), Brasil -

E-mail: luciano.miller@uol.com.br 


\section{Introdução}

O termo espondilolistese degenerativa foi usado por Kilian ${ }^{1}$ em 1854 e é definido como deslizamento ou desvio anterior ou posterior de uma vértebra sobre a outra. $\mathrm{O}$ escorregamento ocorre devido à presença de instabilidade no segmento acometido, geralmente como consequência da doença discal degenerativa, ocasionando sobrecarga nas facetas articulares, evolução com deformidade das articulações e deslizamento vertebral².

O efeito da orientação facetária também foi reportado como fator potencial no desenvolvimento da espondilolistese degenerativa, especialmente quando há inclinação sagital superior a $45^{\circ}$ 3-5.

A espondilolistese degenerativa tem sido considerada uma das principais causas de lombalgia em pacientes acima dos 40 anos e maior fator de estenose do canal vertebral associada à lombociatalgia ${ }^{6-9}$ e que acomete principalmente mulheres, sendo mais comum na região lombar baixa (L4-L5).

Dessa maneira, o objetivo foi avaliar a qualidade de vida dos pacientes portadores de espondilolistese após tratamento cirúrgico.

\section{Método}

Foram selecionados 30 pacientes com espondilolistese degenerativa, do Serviço de Ortopedia e Traumatologia da Faculdade de Medicina do ABC junto ao Hospital Estadual Mário Covas, com indicação de tratamento cirúrgico, no período de janeiro de 2005 a novembro de 2007. Desses, 21 pacientes continuaram o seguimento mínimo por mais de um ano. Foram avaliados 13 homens e 17 mulheres com idades entre 35 e 77 anos.

Como critérios de inclusão, foram utilizadas a presença de espondilolistese degenerativa confirmada por tomografia e ressonância magnética lombar e a falha do tratamento conservador por mais de três meses. Definiu-se para composição dos critérios de exclusão a presença de espondilolistese não-degenerativa, a presença de escoliose acima de $20^{\circ} \mathrm{Cobb}$ e o estudo radiológico incompleto.

Todos os pacientes foram submetidos à avaliação radiográfica (raios X de coluna anteroposterior, perfil, perfil em hiperflexão e hiperextensão) e classificados segundo o grau de deslizamento, de acordo com Meyerding.

A partir daí, foram submetidos a tratamento cirúrgico por via de acesso posterior com dissecção e exposição dos processos espinhosos e facetas articulares bilateralmente. Em seguida, realizou-se a fixação com o uso de parafusos pediculares por meio de parâmetros anatômicos e controle com radioscopia para estabilização in situ da deformidade.

Concluiu-se o procedimento cirúrgico com laminectomia ampla com descompressão central e foraminal, a decorticação do leito de artrodese e a colocação de enxerto autólogo posterolateral.

Profissional ortopedista, que não realizou a cirurgia, entrevistou os pacientes no pré-operatório, pós-operatório em um mês, seis meses e um ano, respectivamente. A escala analógica visual (EVA), o questionário de Roland-Morris e o Índice de Oswestry foram os instrumentos utilizados na avaliação.

Os dados dos pacientes foram expressos em mediana e em valores máximo e mínimo.

Esta pesquisa foi aprovada pelo comitê de ética da FMABC parecer 280/2007.

\section{Resultados}

Segundo a escala analógica de dor, pode-se notar uma melhora significativa em relação à dor nos pacientes submetidos ao procedimento cirúrgico. A pontuação a ela atribuída evoluiu de muito intensa para moderada (Tabela I). Houve melhora da dor em $86 \%$ dos pacientes.

No Índice de Incapacidade de Oswestry, notou-se melhora em mais de $50 \%$ dos casos. Os resultados pós-operatórios foram excelentes em $28 \%$ dos casos e bons em 19\%. Permaneceram inalterados em $48 \%$ dos pacientes e houve piora em apenas $5 \%$ destes. Na avaliação geral, ocorreu uma melhora da capacidade dos pacientes em relação à dor lombar e às atividades da vida diária (Tabela I).

O questionário Roland-Morris de Incapacidade revelou uma melhora progressiva dos pacientes com relação à realização de atividades de rotina. Evidenciou-se uma diminuição de 5,5 pontos de um total de 24 possíveis, ou seja, queda de $22,91 \%$. Os pacientes melhoraram a capacidade de realizar suas atividades rotineiras em $67 \%$ dos casos. Apenas $28 \%$ referiram uma piora do quadro e $5 \%$ permaneceram sem alteração (Tabela I).

Em 70\% dos pacientes a listese ocorreu em L4-L5. O nível L5-S1 foi acometido em $22,86 \%$ dos casos e L3-L4 em apenas 7,14\%.

\section{Discussão}

A espondilolistese degenerativa difere dos outros tipos de deslizamento vertebral devido ao escorregamento relacionado a alterações do disco intervertebral e faceta articular. O nível mais comumente acometido é entre L4-L5, em decorrência da orientação facetária ${ }^{3-5}$. Neste estudo houve concordância em relação ao nível acometido em $70 \%$ dos casos.

Existe um consenso na literatura em relação ao início do tratamento conservador antes de uma indicação cirúrgica ${ }^{10}$. O tratamento

Tabela I - Valores atribuídos por meio dos testes de qualidade de vida aplicados nos pacientes com espondilolistese degenerativa

\begin{tabular}{lcccc}
\hline & Pré-operatório & 1 mês & 6 meses & 1 ano \\
\hline EVA & $9,75(4-10)$ & $6,5(9,5-1,5)$ & $4,5(7-0)$ & $4(9-0)$ \\
RM & $16,5(22-3)$ & $16,5(24-3)$ & $12(20-1)$ & $11(19-1)$ \\
OSW & $60(95-12,5)$ & $68(82,5-26)$ & $46(72,5-10)$ & $42(65-4)$ \\
N & 30 & 22 & 21 & 21 \\
\hline
\end{tabular}

EVA: escala visual analógica; RM: questionário Roland-Morris de incapacidade; OSW: índice de Oswestry; N: número de pacientes. 
deve visar à educação do paciente, orientando-o com relação aos medicamentos para controle da dor, aos exercícios de alongamento e à estabilização da musculatura lombar, juntamente com o trabalho cognitivo. Apesar desse consenso, não foram encontrados na literatura trabalhos que comparem o tempo de tratamento conservador com o resultado cirúrgico desses pacientes.

Comparando o nosso estudo com o de Gaetani et al. ${ }^{11}$, os quais também utilizaram a escala de Roland-Morris para avaliação da melhora da dor, tivemos resultados muito próximos. Nesta avaliação, ocorreu diminuição da dor $(>5$ pontos em $52 \%,<5$ pontos em $14 \%$ e pontuação inalterada ou pior em $34 \%$ ). No estudo prévio houve queda de mais de 5 pontos em $59,4 \%$ dos casos, menos de 5 pontos em $13,1 \%$ e piora ou manutenção da pontuação em 27,5\%. Apesar de muito semelhantes, nossos resultados são um pouco inferiores, possivelmente devido ao tempo de seguimento, pois notamos nos dois trabalhos uma melhora dos resultados com o passar do tempo.
Realizamos fusão em todos os pacientes. Weinstein et al. ${ }^{12}$ publicaram um estudo em que não realizaram artrodese em todos os casos, apresentando um índice de sucesso um pouco inferior ao nosso. Diversos estudos publicados relacionam a artrodese com a melhora da qualidade de vida a longo prazo. No estudo de Gaetani et al. ${ }^{11}$, nos casos em que foi realizada fusão, houve melhora da qualidade de vida.

Os pacientes tiveram melhora muito expressiva da dor após o procedimento cirúrgico, aproximadamente $86 \%$. Booth et al. ${ }^{13}$ relataram índices semelhantes ao nosso estudo com relação à dor.

A espondilolistese com a realização da artrodese posterolateral apresenta uma melhora da qualidade de vida, tornando uma opção de tratamento para os pacientes que não conseguem retornar às suas atividades habituais com a realização de tratamento conservador.

Assim, o tratamento cirúrgico por meio da artrodese posterolateral, associado à descompressão, possibilitou melhora na qualidade de vida dos pacientes com espondilolistese degenerativa.

\section{Referências}

1. Barney L, Freeman III. Escoliose e cifose. In: Canale ST. Cirurgia ortopédica de Campbell. São Paulo: Manole; 2006. p. 1891.

2. Williams KD, Park AL. Lombalgia e transtornos dos discos intervertebrais In: Canale ST. Cirurgia ortopédica de Campbell. São Paulo: Manole; 2006. p. 2093.

3. Boden SD, Riew KD, Yamaguchi K, Branch TP, Schellinger D, Wiesel SW. Orientation of the lumbar facet joints: association with degenerative disc disease. J Bone Joint Surg Am. 1996;78(3):403-11.

4. Grobler LJ, Robertson PA, Novotny JE, Pope MH. Etiology of spondylolisthesis: assesment of the role played by lumbar facet joint morphology. Spine. 1993;18(1):80-91.

5. Love TW, Fagan AB, Fraser RD. Degenerative spondylolisthesis. Developmental or acquired? J Bone Joint Surg Br. 1999;81(4):670-4.

6. Anderson GBJ. Epidemiology of spinal stenosis. In: Weinstein JN, Gordon SL, editors. Low back pain: a scientific and clinical overview. Rosemont: American Academy of Orthopaedic Surgens; 1996 p. 637-41.
7. Friberg 0. Instability in spondylolisthesis. Orthopedics. 1991;14(4):463-5

8. Frymoyer JW. Degenerative spondylolisthesis: diagnosis and treatment. J Am Acad Orthop Surg. 1994;2(1):9-15.

9. Herkowitz HN. Spine update. Degenerative lumbar spondylolisthesis. Spine (Phila Pa 1976). 1995;20(9):1084-90.

10. Kalichman L, Hunter DJ. Diagnosis and conservative management of degenerative lumbar spondylolisthesis. Eur Spine J. 2008;17(3):327-35.

11. Gaetani P, Aimar E, Panella L, Levi D, Tancioni F, Di leva A, et al. Functional disability after instrumented stabilization in lumbar degenerative spondylolisthesis: a follow-up study. Funct Neurol. 2006;21(1):31-7.

12. Weinstein JN, Lurie JD, Tosteson TD, Hanscom B, Tosteson AN, Blood $E A$, et al. Surgical versus nonsurgical treatment for lumbar degenerative spondylolisthesis. N Engl J Med. 2007;356(22):2257-70.

13. Booth KC, Bridwell KH, Eisenberg BA, Baldus CR, Lenke LG. Minimum 5-year results of degenerative spondylolisthesis treated with decompression and instrumented posterior fusion. Spine (Phila Pa 1976). 1999;24(16):1721-7. 